\title{
CONSORCIO REGIONAL DE TRANSPORTE DE MADRID Y EMPRESA TRANSMILENIO DE BOGOTÁ
}

\author{
Ramiro Andrés Rodríguez Otavo \\ Escuela de Negocios, Gestión y Sostenibilidad \\ Programa Administración de Empresas \\ rarodriguezot@poli.edu.co \\ Sebastián Vargas \\ Escuela de Negocios, Gestión y Sostenibilidad \\ Programa Administración de Empresas \\ svargas@poli.edu.co \\ Allynson Karen Venegas Camargo \\ Docente Programa de Administración de Empresas \\ vcallynson@poligran.edu.co
}

\section{Resumen}

Este articulo ahonda sobre el transporte público en Madrid España versus Bogotá Colombia. Muestra cómo funciona, sus rutas y estructura del transporte público en cada una de las capitales, a su vez nos brinda estadísticas de movilidad en ambas partes e identifica la situación actual y cómo ha evolucionado el transporte con el pasar de los años.

Finalmente resaltamos que podemos aprender de cada una, a su vez analizamos desde el punto de vista social que impacto genera en la ciudadanía un mejor transporte, más accesibilidad y un mejoramiento en las tarifas para los diferentes públicos.

Palabras clave: Transporte; movilidad, evolución, ciudadanía, mejoramiento.

\section{Introducción}


Todas las comunidades cuentan con un medio de transporte público o privado con el objetivo de acortar distancias y lograr ser más eficientes en sus actividades diarias, principalmente en las grandes urbes del mundo es de suma importancia ya que se convierte en una arteria fundamental para su dinamismo social, cultural y económico.

Partiendo de esta idea, en la historia se ha buscado desarrollar múltiples transportes que se adapten a las distintas culturas, que permitan de esta manera ser más eficientes en los distintos retos. Las ciudades buscan innovar en grandes sistemas que permitan la conectividad entre ellas, y de esta manera ser reconocida como ejemplo.

Sin embargo, cabe resaltar que toda esta implementación viene atado a una serie de variables que pueden ser cruciales al momento de tomar decisiones a futuro que podrían presentar fallas en el sistema hasta el punto de provocar un colapso en la ciudad.

Entre otros, los sistemas de transporte son los buses, los metros, los tranvías que podemos encontrar en la actualidad en varias ciudades del mundo. Esto a su vez viene acompañado de una adecuada malla vial, la cual es vital para el buen funcionamiento de los diferentes medios de transporte o un buen sistema eléctrico en el caso de los metros, los ferris o los tranvías. Sin dejar de lado la red de semaforización en la ciudad, tan nombrada últimamente en la capital colombiana de acuerdo con distintos medios de comunicación local como El tiempo, sin embargo, muchos de los problemas que pueden tener en la actualidad estos transportes, están relacionados con los mismos ciudadanos, como es el caso de Bogotá. Una ciudad en la que carece de identidad al adoptar ideologías fallidas y resultan destruyendo lo que realmente somos.

En esta oportunidad el objetivo es ver las diferencias entre los sistemas de transporte público entre las ciudades de Bogotá en Colombia y Madrid en España. Cabe resaltar que aun estando lejos entre ellas, Bogotá principalmente se ha visto muy influenciada por la cultura española desde sus inicios y el transporte público no es la excepción, ya que se han realizado consultorías en este tema con autoridades de este país.

Comencemos con la capital española; Madrid es una ciudad con más de seis millones habitantes y es una de las 17 comunidades autónomas de España de acuerdo con la información suministrada al 2016 por el instituto nacional de estadísticas de España, su territorio se puede dividir en tres grandes partes, dependiendo su influencia y la mayor concentración de la población y su economía. Estas zonas las podemos reconocer como la zona A, esta zona reconocida por ser el centro de la ciudad con la mayor concentración de habitantes, seguidamente encontramos las coronas B que, si bien están en una zona de influencia bastante concurrida por los madrileños, tiene menos población que la zona A, por 
último, encontramos las coronas $\mathrm{C}$ reconocidas por ser poblaciones aledañas con un ritmo de vida mucho más tranquilo que el de las demás zonas. Identificando que más de $50 \%$ de la población está en la zona central de la ciudad, con aproximadamente $3.4 \mathrm{M}$ de habitantes, tomando los datos de la página oficial de la comunidad de Madrid.

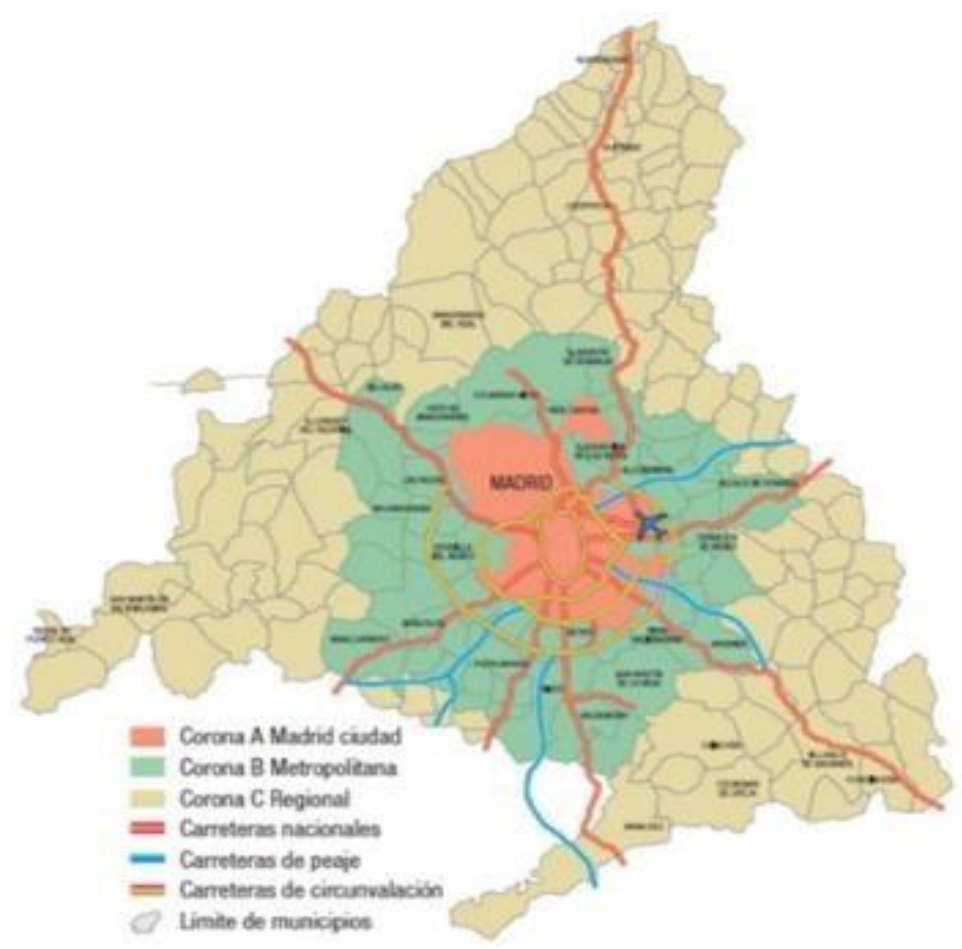

Ilustración 3: Estructura Territorial; Madrid

Fuente: Citram, 2016

Según estudio del consorcio regional de transportes de Madrid, en el año 2015 dentro de toda la comunidad de Madrid se realizaron 1.385,7 millones de viajes independientemente del tipo de transporte que use la persona. Se logra evidenciar que para los madrileños es igual de beneficioso caminar para viajes cortos, tomar el transporte público para los viajes un poco más largos y el transporte privado para viajes más largos. Esta afirmación se hace con base en el estudio realizado por la misma entidad (CRTM) donde se ve que estas tres modalidades están ponderadas en porcentajes muy parecidos (32\% aprox.)

Dicho esto, el sistema de transporte de Madrid tiene seis distintas opciones que se articulan para poder prestar un servicio que abarque las distintas zonas ya mencionadas. Entre estos encontramos el Metro de Madrid, Autobuses urbanos en la ciudad de Madrid, autobuses urbanos en otros municipios, autobuses interurbanos, ferrocarril de cercanías (Renfe) y el 
metro ligero. Las zonas tarifarias van directamente relacionadas a las zonas de cobertura (A, B y C), inclusive existe la posibilidad de ir a otras zonas aledañas enmarcadas como la $\mathrm{E}$, en las que se puede ir a las zonas de Castilla - La mancha.

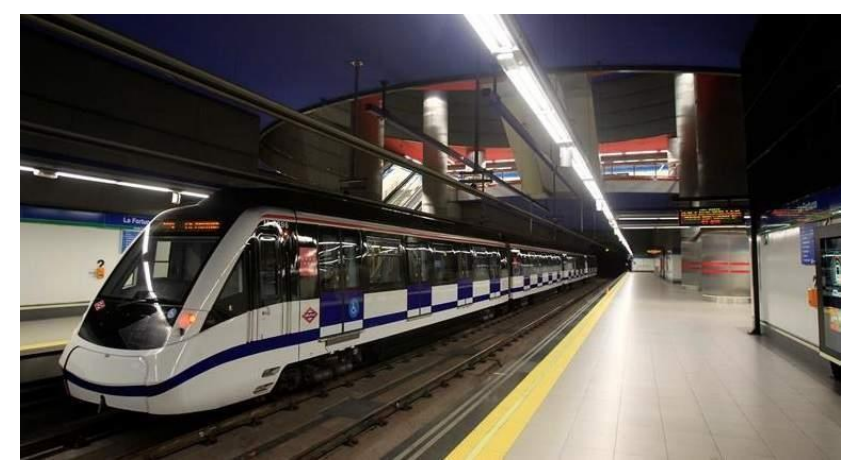

Ilustración 4: Metro de Madrid

Fuente: El Economista, 2017

De acuerdo con las fichas históricas del ayuntamiento de Madrid podemos encontrar que el funcionamiento del transporte público está a cargo de una entidad llamada Consorcio Regional de transportes de Madrid (CRTM) fundada en el año 1985 al mando de entidades estatales, dichas entidades son el ayuntamiento de Madrid y la comunidad de Madrid con la necesidad de proporcionar un sistema integrado de transporte público, sin embargo, el sistema llamado Renfe que también está conectado al mismo sistema está a cargo del gobierno español, ya que a diferencia de los demás opera en distintos lugares con el objetivo de conectar a Madrid con las demás comunidades autónomas. CRTM a su vez contratan empresas privadas que trabajan como operadores de varios de los métodos de transporte. Por ejemplo, el operador del metro de Madrid es una concesión con una empresa privada a 30 años llamada Transportes Ferroviarios de Madrid, para los buses urbanos y los interurbanos opera el mismo modelo. Como su objetivo es manejar una integralidad en todo aspecto, sucede lo mismo con los sistemas de pago o a integración tarifaria la cual busca manejar un solo método de transacción para todos los transportes. De igual forma ofrece subsidios dependiendo la población a la que va dirigida como las personas menores de 26 años al poder viajar ilimitadamente por 20 euros al mes. De igual forma hay gran variedad de boletos o abono que ofrecen con el objetivo de cumplir las necesidades del cliente. De acuerdo con el último informe anual del CRTM hecho en el 2015, para el año 2018 quedara obsoleto el funcionamiento de los boletos magnéticos y entraran en vigor las nuevas tarjetas recargables con los pasajes necesarios y las zonas que más se frecuentan, facilitando los accesos al mismo. Sumado a esto claro está la integración modal con los transportes.

Existen intercambiadores de transporte en la región de Madrid, ubicados estratégicamente buscando hacer el trasbordo de pasajeros entre los buses urbanos e interurbanos. El primero 
llamado Aluche fue construido en 1986 en la época en la que se movía aproximadamente 12 millones de pasajeros por año, para el año de 1995 se construyó Moncloa ya que se triplico la demanda con 60 millones de pasajeros al año, y para el año 1998 con 113 millones de viajes se construyó lo el intercambiador de américa. Seguido esto entre los años de 2003 y 2007 se realizaron concesiones para la construcción de cuatro nuevos intercambiadores que son plaza castilla, Moncloa, príncipe pio y plaza elíptica.

Además, el sistema de buses de Madrid busca un modelo de transporte sostenible, al enfocarse en la circulación de este tipo de transporte en las zonas industriales en las que anteriormente solo se podía lograr el acceso con automóvil particular. De esta manera transporta a los trabajadores hasta conexiones que tenga con el metro de Madrid.

En la oferta de transporte que la comunidad de Madrid ofrece a sus visitantes y residentes encontramos:

\begin{tabular}{|c|c|c|c|c|c|}
\hline $\begin{array}{l}\text { Operadores de } \\
\text { Transporte }\end{array}$ & $\begin{array}{l}\text { Metro de } \\
\text { Madrid }\end{array}$ & $\begin{array}{c}\text { Autobús } \\
\text { urbano } \\
\text { de Madrid } \\
\text { (EMT) }\end{array}$ & $\begin{array}{c}\text { Concesiones } \\
\text { por } \\
\text { carretera }\end{array}$ & $\begin{array}{l}\text { Metros } \\
\text { ligeros } \\
\text { y otras } \\
\text { concesiones } \\
\text { ferroviarias }\end{array}$ & $\begin{array}{l}\text { Renfe- } \\
\text { Cercanías }\end{array}$ \\
\hline No de líneas & 12+Ramal & 204 & 439 & 5 & 9 \\
\hline Longitud-red (km) & 269,5 & $1.533,7$ & $8.447,0$ & 54,8 & 391,0 \\
\hline Longitud-líneas ( $\mathrm{km}$ ) & 269,5 & $3.622,8$ & $20.843,0$ & 54,8 & 778,3 \\
\hline $\begin{array}{l}\text { No de estaciones/ } \\
\text { paradas-red }\end{array}$ & 236 & 4.660 & 8.083 & 62 & 94 \\
\hline $\begin{array}{l}\text { No de estaciones/ } \\
\text { paradas-líneas }\end{array}$ & 286 & 10.706 & 20.951 & 63 & 169 \\
\hline $\begin{array}{l}\text { No vehículos } \\
\text { (coches/ buses) }\end{array}$ & 2.320 & 1.908 & 1.810 & 241 & 1.127 \\
\hline Coches-km (millones) & 165,3 & 87,8 & 172,0 & 16,1 & 145,3 \\
\hline
\end{tabular}

Ilustración: oferta de transporte

Fuente: Metro de Madrid

Metro: Tren urbano con capacidad masiva y de frecuencia constante Transporte Publico en Bogotá y Madrid.

Metro ligero: Capacidad intermedia de transporte con frecuencia regular y de característica interregional. 
- Bus: transporte público para un número limitado de personas con trayectos fijos, con circulación en vías urbanas.

- Tren: Transporte principalmente para trayectos largos, con gran flujo de personas. Identificado por conectar gran número de vagones. Según información oficial del gobierno español. El metro de Madrid dispone de 12 líneas que conectan la ciudad más un ramal que conecta las estaciones de príncipe pio y la estación de ópera, es considerada la 3 más importante del mundo debido a su capacidad al abarcar 294km con 289 estaciones. El EMT es la empresa encargada de manejar la red de autobuses urbanos de la ciudad de Madrid abarcando gran parte de la ciudad en la que no cuentan con una línea de metro cerca o simplemente como otra opción de transporte en la ciudad de Madrid, se comprende por 204 líneas en las que encontramos 177 diurnas y 26 nocturnas. La única que encontramos trabajando 24/7 es la ruta con destino al aeropuerto. Del mismo modo, encontramos los buses interurbanos, en los que destacamos la conexión de Madrid con las comunidades cercanas, debido a sus recorridos, dobla en cifras los buses urbanos de Madrid, teniendo más de 400 buses a disposición de los madrileños y aledaños. Seguidamente encontramos el Renfe tren de cercanías, utilizado principalmente como método de transporte entre las distintas poblaciones de la comunidad autónoma de Madrid, y como método de conexión entre los demás sistemas férreos que comprenden el país español.

TRANSPORTE DE VIAJEROS POR CARRETERA

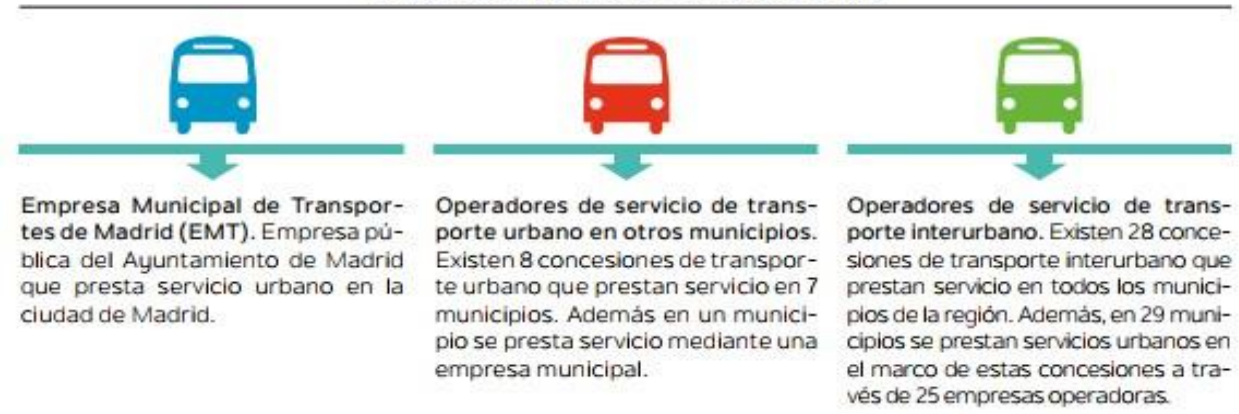

TRANSPORTE DE VIAJEROS POR FERROCARRIL

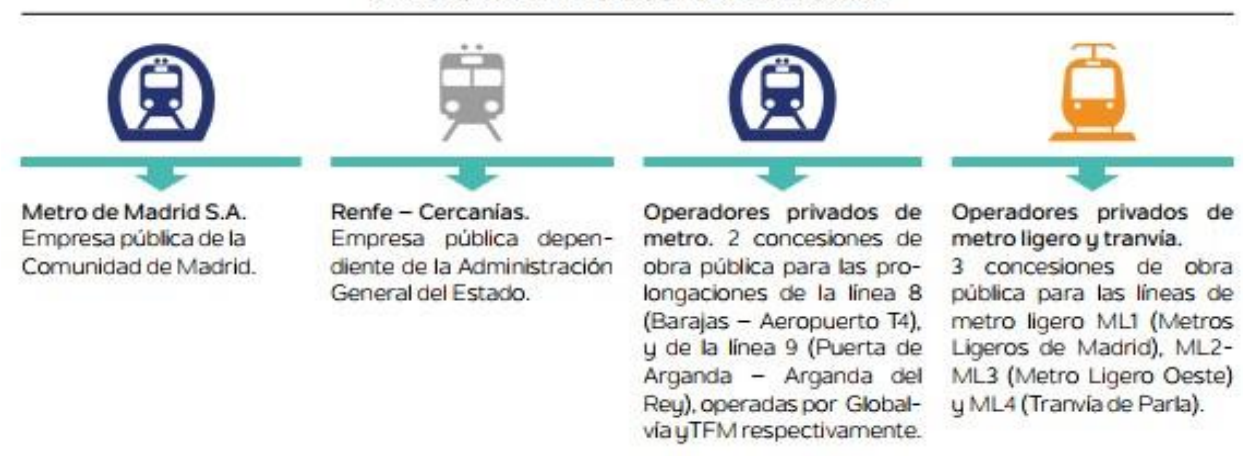

Grafico 2: Consorcio regional de transporte de Madrid, 2015 
Al momento de operan mancomunadamente los distintos sistemas de transporte, se cuenta con un centro de cómputo que ofrece la habilidad articular los tiempos y funcionamientos de estos. Esto lo realizan por medio de CITRAM centro de innovación y gestión de la movilidad del consorcio regional de transportes de Madrid. Esta entidad actúa como cerebro del transporte, ya que supervisa al instante los movimientos que este pueda tener y solucionar en tiempo real. De igual forma recibe información de cada empresa para poder adaptar un solo modelo de gestión y poder brindar dicho soporte, siendo unos de los pioneros en este modelo y tomado como ejemplo a nivel de consultoría. En cuanto a información suministrada en el año 2014 se demuestran cifras en su estado de pérdidas y ganancias por $\$ 1.899$ millones de euros en ingresos y $\$ 1.869$ millones en gastos, dejando una utilidad en el año por $\$ 31.6$ millones de euros.

Bogotá, Colombia un país que actualmente cuenta con 49.547.962 habitantes, de los cuales 8.080.734 están ubicados en Bogotá según el DANE (2016) y con el pésimo diseño de transporte en Bogotá en los años 90 donde generan una sobre oferta en el servicio de transporte público en la capital según Revista de Ingeniería, (29), 156-162., encontramos que tras varios años de estudio podemos identificar que la situación de la capital en temas de movilidad ha tenido un mejoramiento pero no en la medida que lo necesita, ya que vemos que el sistema actual Transmilenio y SITP aún no cubre la demanda solicitada ni en servicio, ni en calidad ni en infraestructura, cada vez más necesitamos más vías públicas que soporten el peso de los buses tanto alimentadores, complementarios o buses normales y que contribuyan al mejoramiento del transporte público y privado del país, con el paso del tiempo la población incrementa y el sistema empeora, un ejemplo para demostrarlo es cuando llueve la ciudad colapsa en movilidad, esto hace que la falta de infraestructura, vías y un sistema bien diseñado en transporte público genere caos. Otro punto que interfiere es la administración que ejerce el estado en el sistema ya que pese a tener un sistema (Transmilenio) bien diseñado hay factores que influyen en el desarrollo de este perjudicando la movilidad y sumado a esto está la mala administración y la creciente corrupción y carruseles que salpica al país en todos sus frentes públicos según la revista Semana 2016, esto hace que haya menos inversión en trasporte, vías públicas e infraestructura para mejorar el trasporte púbico en Bogotá. También debemos analizar que Bogotá ha tenido un crecimiento poblacional en los últimos años, el cual es generado por la presencia de individuos de otras ciudades que llegan en busca de oportunidades, y de crecimiento". El crecimiento poblacional es un punto de suma importancia ya que dé también del crecimiento poblacional el cual ha incrementado en un $9 \%$ por año según estadísticas del Dane. 


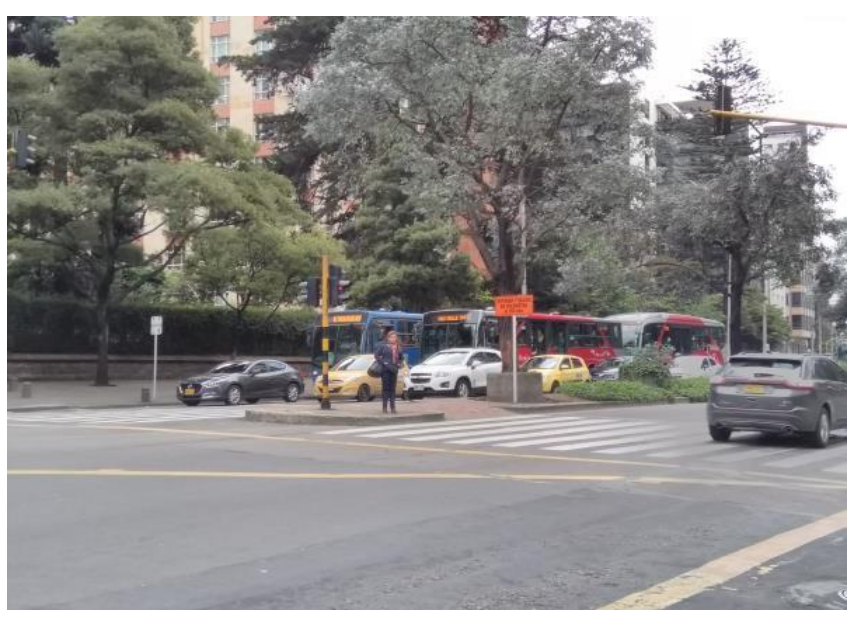

Ilustración 5: Transmilenio -

Andres Rodriguez, Otavo, 2018

Como comenta Aparicio (2010, p. 33-64): “el crecimiento y expansión de las ciudades durante el último siglo no puede ser visto sin contar con el importante papel que han asumido las infraestructuras del transporte. En Bogotá, el paso por el tranvía, los buses y el automóvil ha sido determinante, pues cada una de estas etapas marcó para la ciudad cambios drásticos en su morfología este depende en cierta medida el transporte en la capital, esto dado que a medida que la población va creciendo ya se en volumen de personas o de turistas que se radiquen en la ciudad se deben tomar medidas que permitan mejorar la movilidad en Bogotá puesto que si ahora es un caos en un futuro será peor. En otros estudios realizados existen autores que critican el modelo de trasporte de Bogotá "las deficiencias serias del modelo Transmilenio para los pasajeros: el sistema es relativamente lento, muy costoso, produce exclusión social y no contribuye al cumplimiento de los Objetivos de Desarrollo del Milenio" (Moller, 2010, p. 38-39). Otro aspecto importante a tratar en este documento es el tema de la cultura ciudadana en cuanto transporte público se trata ya que día a día vemos como los ciudadanos no respetan las normas dispuestas por Transmilenio al no colarse y pagar el pasaje para poder transportarse, también vemos a múltiples vendedores ambulantes vendiendo productos sin ningún tipo de garantía ni control; esto hace que el sistema pierda calidad y que sea un trasporte cómodo para la gente que viaja por trabajo o de ocio. Estas dos variables se suman y se congestiona el sistema en todos los horarios y más aún en horas pico donde el grueso de la ciudad se dirige hacia sus casas. 


\section{Pasajeros transportados y variación anual \\ I trimestre de 2012 - III trimestre 2017p}

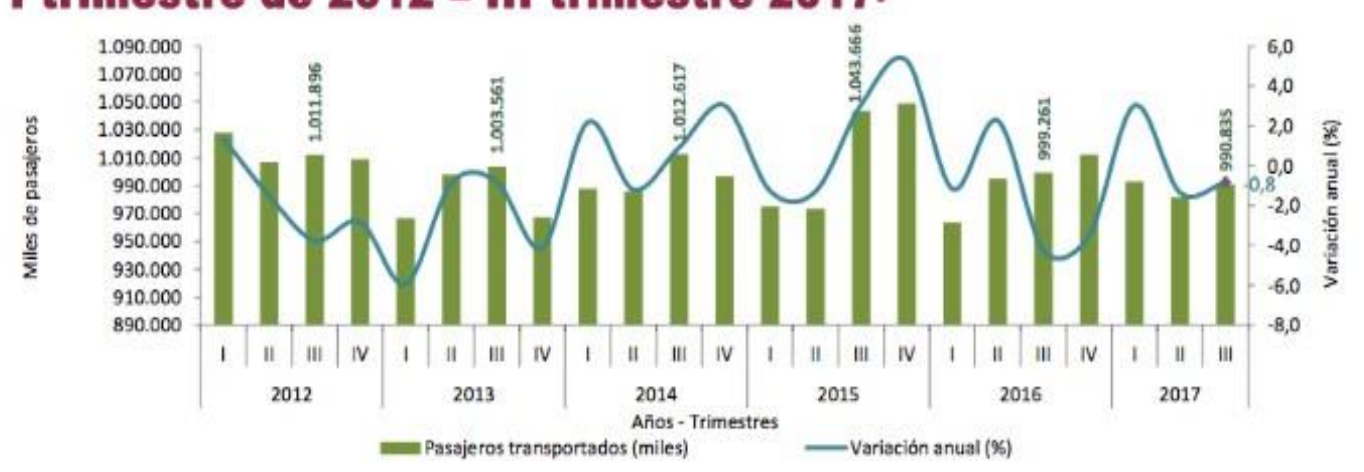

Fuento: DANE - ETUP p Cifra provisional

\section{Grafico 3: Pasajeros Transportados}

Fuente: Dane (2017)

Si bien es cierto el transporte en Bogotá no es ejemplar como en otras capitales del movilizarnos desde puntos lejanos por ejemplo de Norte a Sur en 30 minutos máximo 40 minutos, pero si lo comparamos con los buses tradicionales nos demoramos entre una hora y 20 minutos máximo 1 hora y 30 minutos este genera ahorro en tiempos de cada persona y en cierta medida una calidad de servicio. También cuenta con una única tarjeta de pago que sirve para los diferentes transportes como son TM, SITP y buses complementarios, además de esto esta tarjeta se puede personalizar, primero para mundo, pero si tiene puntos positivos en cuanto a movilidad ya que nos permite no pagar los transbordos que se realicen en tiempos determinados y segundo para no perder el saldo que tenga y poder recuperarlo. Otro punto positivo es que los buses para el transporte masivo (Transmilenio) están en buen estado en comparación a otro tipo de buses que hay dispuestos para en transporte público y en su mayoría permanecen limpios y tienen mantenimiento preventivo, esto hace que sea seguro y preste un buen servicio.

Pese a lo anterior el sistema de transporte en Bogotá no basta con tener un sistema de transporte integrado y que sea eficiente en tiempos, debemos concientizarnos y buscar una nueva herramienta para movilizarnos y la opción más acertada sería el metro de Bogotá que podría ser subterráneo o elevado. Según los estudios realizados por la administración actual de Bogotá encuentran que crear el metro elevado es la mejor opción dado los más de 31 indicadores evaluados (ambientales, urbanos, constructivos, sociales, financieros y de riesgos) donde evidencian que, para Bogotá dada la estructura, los suelos y el factor económico es la mejor opción para mejorar la movilidad en la 
capital. El factor económico tuvo el mayor peso y más importancia dado los indicadores presentados por el metro de Bogotá:

Con lo que se construye un kilómetro de metro subterráneo se puede construir 1,48 kilómetros de metro elevado. Genera ahorros de inversión US 61 millones de dólares por cada kilómetro.

El costo de operación del metro elevado es $28 \%$ menor que el subterráneo. No requiere ventilación mecanizada, ni bombeo para extracción de aguas y solo iluminación nocturna Según metro Bogotá, "En el año 2016, el gobierno nacional y distrital impulsaron un estudio de alternativas, tipologías y costos a cargo de la empresa SYSTRA, filial del Metro de París. En el ejercicio de análisis, Systra evaluó y comparó ocho opciones de longitudes de trazado, con tramos elevados y bajo tierra, en este ejercicio, el mayor peso relativo lo tuvo el componente económico, donde se encontró que:

- Con lo que se construye un kilómetro de metro subterráneo se puede construir 1,48 kilómetros de metro elevado. Genera ahorros de inversión US 61 millones de dólares por cada kilómetro.

- El costo de operación del metro elevado es 28\% menor que el subterráneo. No requiere ventilación mecanizada, ni bombeo para extracción de aguas y solo iluminación nocturna.

- El metro elevado se puede construir más rápido porque las vigas se pueden prefabricar en un taller y luego se instalan rápidamente sobre las columnas, incluyendo las estaciones. La construcción de $20 \mathrm{~km}$ elevados permite reducir los tiempos de obra de 66 a 40 meses. Los tiempos de instalaciones electromecánicas son similares y añaden seis meses al programa de obra.

- La obra del metro elevado presenta menores riesgos constructivos en Bogotá. Los riesgos valorados de la solución subterránea se relacionan con la excavación por debajo de cuerpos de agua y la desecación del suelo, que en Bogotá produciría asentamientos en las edificaciones hasta a varias cuadras de distancia.

- Los riesgos operativos también fueron considerados. En trenes bajo tierra es más complejo el manejo de emergencias tales como incendios, atentados o accidentes y trenes varados." Después de generar estos estudios y para lograr esto y corregir todas las falencias que tiene el transporte y que permea varias situación del país es necesario hacer un replanteamiento en la administración distrital en cuanto a los recursos que se destinan para este tipo de inversiones porque actualmente la corrupción no da tregua ya que en casi un $60 \%$ de los dirigentes resulta salpicado por algún tipo de carrusel, mientras esto no se solucione hay muchas cosas que no se podrán mejorar como por ejemplo la movilidad ámbito que es supremamente importante para dar calidad de vida a los ciudadanos que residen en la capital. También hay que reinventar la educación y la cultura en el país ya que sin estos dos pilares no hay estructura o estrategia que valga porque si no respetamos a los demás, no respetamos la fila o pagar un pasaje sencillamente seria supremamente difícil 
lograr que el país un avance y en el término de un par de años sea ejemplar para otros países y culturas. Teniendo en cuenta lo analizado anteriormente debemos hacer una reflexión acerca del transporte público entendiendo que no es solamente la infraestructura, vías, gobernantes, sino que también depende de nosotros mismos es decir que esto también empieza por nosotros mismos, al instruirnos e ir más allá de lo que nos brindan los dirigentes y los medios de comunicación porque es importante estar informado para así forjar nuestro criterio con diferentes puntos de vista.

\section{Ámbito social}

Retomando y dándole una mirada más detenida al ámbito social, entendiendo que el transporte público va más allá de tener un transporte organizado, seguro y de calidad, se debe impartir una movilidad accesible para todo tipo de públicos. Generalmente las personas que más dan uso al transporte público son las personas de estratos uno, dos y tres, estas personas a nivel socioeconómico son las menos favorecidas ya que mensualmente obtienen como ingreso un poco más del salario mínimo legal vigente y sus gastos con el pasar del tiempo ascienden cada vez más.

Teniendo en cuenta lo anterior y que tenemos el transporte público más caro de Latinoamérica superado únicamente por Santiago de Chile y Rio de Janeiro, evidenciamos una problemática preocupante debido a que si la mayoría de las personas que utilizan el transporte público son de escasos recursos y por donde miremos; frecuencia del servicio, tiempos de espera, tarifas, accesibilidad y calidad del servicio hace que sea una movilidad caótica, entonces ¿Cómo se logra que la gente de a pie pague por este servicio?.

\begin{tabular}{|r|c|c|}
\hline \multicolumn{3}{|c|}{ INGRESO SALARIO MINIMO LEGAL VIGENTE VS TARIFA } \\
TRANSPORTE PÚBLICO
\end{tabular}

Grafico 4: Ingresos de Familias vs Tarifa Autoría propia y datos DANE y Transmilenio S.A, 2016 
De la anterior gráfica podemos deducir que no es coherente la inflación que ha tenido el costo del pasaje con relación al incremento anual del salario mínimo en Colombia, esto debido a que en promedio el valor gastado mensual es de $\$ 87.500$ pesos el cual representa un $18 \%$ del salario mínimo el cual es uno de los más bajos en Latinoamérica dado que cubre únicamente el 60\% de la canasta familiar según (Financial Red 2017), por otro lado el transporte sigue siendo el más costoso de Latinoamérica y menos eficaz según revista Semana (2016.)

Una manera de poder sufragar esta problemática seria implantar una diferenciación de tarifas bien estructurada para los adultos mayores, estudiantes y personas sin empleo que le permita ser accesible a todas las personas y a su vez sea sostenible para la empresa prestadora del servicio. Esto no es algo nuevo ya que en varios países del mundo lo practican van desde Estados Unidos hasta el continente europeo. Por ejemplo "El metro de Brasil es el caso más reconocido a nivel latinoamericano, cuenta con tarifas diferenciales para discapacitados, estudiantes, desempleados, adultos de la tercera edad, entre otros. Recientemente en Argentina en la Provincia de Buenos Aires, se logró la tarifa diferencial para los estudiantes, los cuales contaran con el boleto educativo gratuito.” (Transporte Público y desigualdades sociales en Bogotá 2017.)

\section{MOVILIDAD}

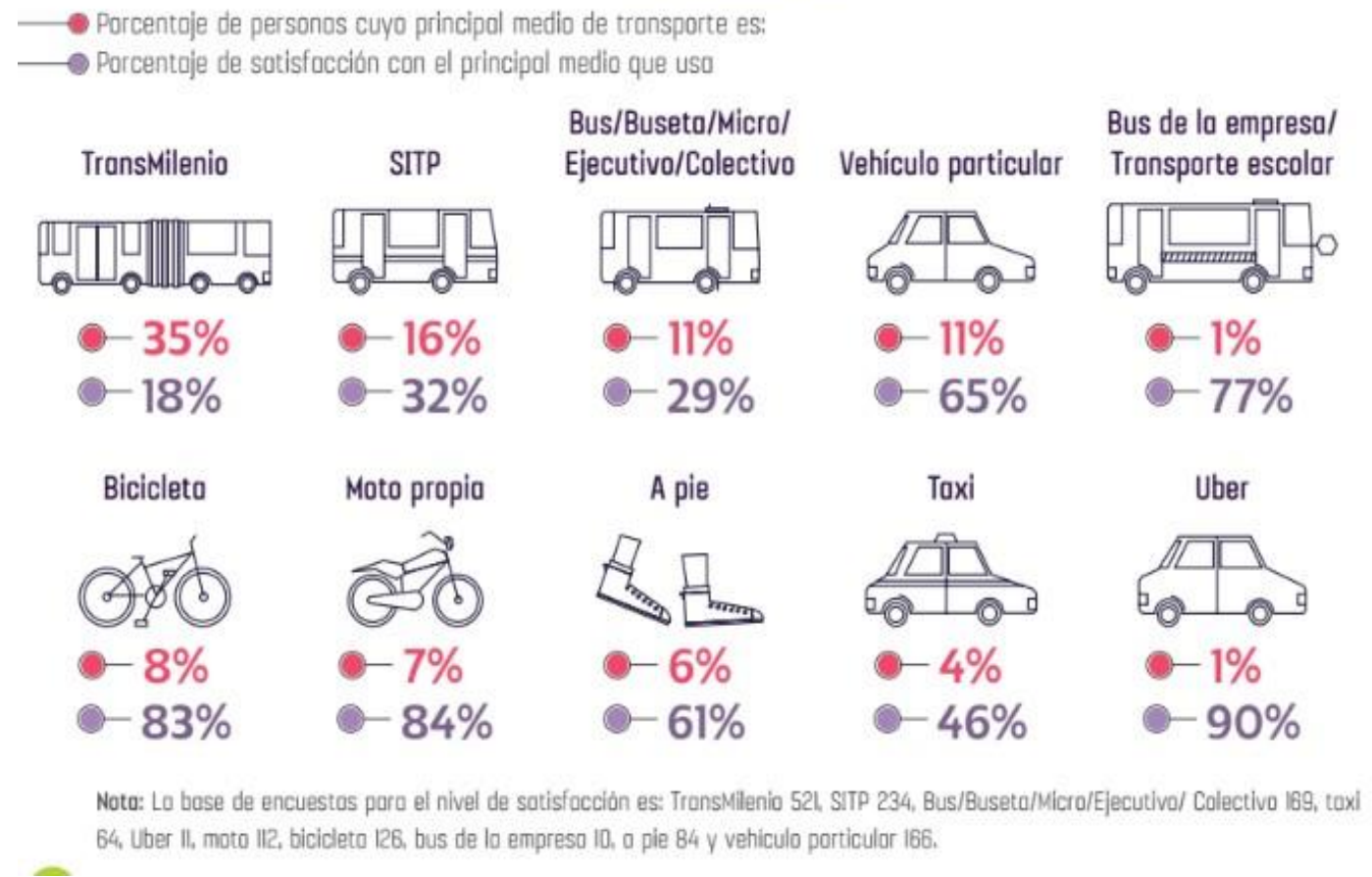

Gráfico 5: Tipos de Transporte - Encuesta de percepción ciudadana 2017. 
Dicha idea sería un buen elemento para luchar en el mejoramiento de la movilidad en la capital y a su vez generar conciencia, aceptación y accesibilidad para los diferentes públicos que se movilizan diariamente por la ciudad. Esto entendiendo que "no puede entenderse el sistema de transporte sin tener en cuenta sus relaciones con la tecnología, el desarrollo económico, la estructura social, las estructuras de poder y el sistema cultural" (Consorcio de Investigación Reactiva, 2010). Por lo tanto, la perspectiva social es de suma importancia para entender y mejorar el transporte en la capital abriendo más oportunidades a los menos favorecidos y erradicando la exclusión social en el acceso a la movilidad. La movilidad en Bogotá debería que ser intrínseca asumiendo su papel de capital ya que por ello llegan cientos de personas de diferentes partes de Colombia, incluso extranjeros a hacer uso del transporte de manera turística o formal como la mayoría. La movilidad debe ser igualitaria ya que hay zonas del país donde se facilita la movilidad como existen partes que no y esto hace que el servicio sea excluyente y tenga cierto grado de inequidad como lo afirma "la movilidad debe entenderse como un derecho, el derecho de todo el mundo a ir a todas partes, un derecho inherente a la condición de ciudadano. No poder ejercerlo conduce, a la inmovilidad, que no es otra cosa que la imposibilidad de acceder a los bienes y servicios de la ciudad, debido al alto costo del transporte" (Dangond Gibsone, Jolly, Monteolova Vilches, \& Rojas Parra, 2011, pág.)

Aspectos Técnicos Cabe resaltar que Bogotá hace parte de las ciudades en el mundo que buscan estar a la vanguardia en el desarrollo de infraestructura urbana que permita articular los factores socio económicos de la ciudad con el objetivo de tener un territorio ordenado de la mano del sistema de transporte público y la comisión de ordenamiento territorial. En este contexto, hay que identificar que en las grandes ciudades del mundo las actividades realizadas por los ciudadanos tienden a localizarse en puntos estratégico de la ciudad los cuales son vitales al momento de tomar algún tipo de decisión en el factor de movilidad. Estos puntos son los encargados de oriental la planeación en temas de oferta y demanda de buses en horarios específicos los cuales permitan tener un equilibrio en el sistema de transporte público. Es por esto por lo que al no cumplir con este equilibrio se pueden presentar fenómenos como el alza de los precios en el servicio o el aumento del tiempo en los recorridos. 


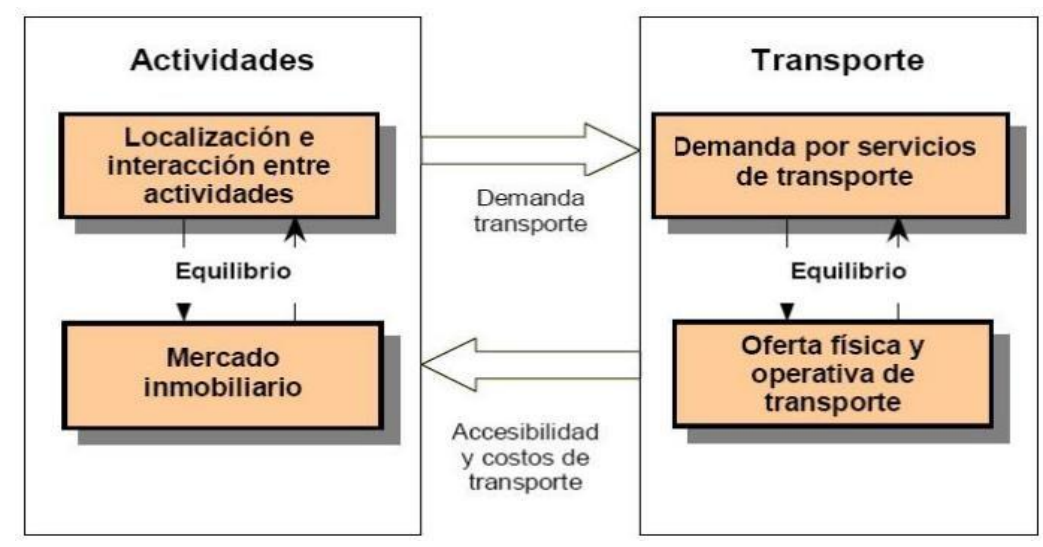

Grafico 6: Sistema de simulación integrado de localización de actividades y transporte

Fuente. Tranus.(2008)

En una manera más simple de entender, podemos decir que los sistemas de transporte publico deben de ir orientados a satisfacer la demanda de una manera en la que aumenten la frecuencia en las zonas que presentan gran flujo de personas como es el caso de los centros financieros, centros empresariales, zonas industriales y zonas de alto comercio.

Además, podemos entender el sistema integrado de transporte como uno de los desarrollos urbanísticos más importantes para cada ciudad ya que dependen considerablemente de los planes de desarrollo territorial establecidos. Cabe destacar que el mal uso del suelo es lo que provoca desorden a nivel estructural como es el caso los embotellamientos, exceso de contaminación y densificación de las zonas populares. Al presentarse este tipo de problemas, es cuando el ciudadano opta por utilizar un modelo de transporte que se ajuste a las condiciones que le ofrezca la ciudad.

El pensar en una ciudad como Bogotá, podemos darnos cuenta de los grandes problemas estructurales que presenta desde sus inicios, lo que ha conllevado a la incrementar la dependencia frente al carro privado o familiar. Como ejemplo encontramos las urbanizaciones dispersas o en ocasiones en la periferia de la ciudad, por otro lado, encontramos el estímulo cultural de realizar actividades de ocio en recintos que estimulen el uso del carro, como las visitas a los centros comerciales. De igual forma la invasión del espacio público y la sensación de inseguridad. Contrario a esto se debe proponer un desarrollo urbanístico de inclusión con el peatón en el que estimule los transportes alternos como mecanismo de movilidad, espacios públicos más seguros y con una oferta cultural variada que ofrezca la una armonía urbanística con las zonas residenciales. 


\begin{tabular}{|c|c|c|}
\hline ATRIBUTO & EXPANSION & CRECIMIENTO INTELIGENTE \\
\hline Densidad & Baja densidad & Alta densidad \\
\hline $\begin{array}{c}\text { Característica del } \\
\text { crecimiento }\end{array}$ & $\begin{array}{c}\text { Desarrollo en la periferia urbana (en } \\
\text { los espacios verdes) }\end{array}$ & $\begin{array}{c}\text { Desarrollo (o relleno) de las zonas } \\
\text { urbanas }\end{array}$ \\
\hline $\begin{array}{l}\text { Mezcla del uso de los } \\
\text { suelos }\end{array}$ & Usos del suelo homogéneos & Mezcla de los usos del suelo \\
\hline Escala & $\begin{array}{c}\text { Grandes edificios y construcciones, } \\
\text { vias anchas. Poco detalle ya que la } \\
\text { gente aprecia el paisaje desde lo } \\
\text { lejos, siendo la mayoria conductores. }\end{array}$ & $\begin{array}{c}\text { Escala humana. Edificios, } \\
\text { construcciones y vias más } \\
\text { pequeños. Más detalle en el } \\
\text { cuidado y el diseño de } \\
\text { construcciones para peatones. }\end{array}$ \\
\hline Transporte & $\begin{array}{l}\text { Orientado hacia el uso del automóvil. } \\
\text { Poco conveniente para los peatones. } \\
\text { ciclistas y el transporte público. }\end{array}$ & $\begin{array}{l}\text { Multi-modal. Apoya el transporte } \\
\text { público, los ciclistas y los } \\
\text { peatones. }\end{array}$ \\
\hline Diseño de las vias & $\begin{array}{c}\text { Vías diseñadas para maximizar el } \\
\text { tráfico y las velocidades del } \\
\text { automóvil. }\end{array}$ & $\begin{array}{c}\text { Vias diseñadas para satisfacer } \\
\text { diferentes actividades y calmar el } \\
\text { tráfico. }\end{array}$ \\
\hline $\begin{array}{l}\text { Proceso de } \\
\text { planeación }\end{array}$ & $\begin{array}{l}\text { Poca planeación y coordinación entre } \\
\text { jurisdicciones y empresarios. }\end{array}$ & $\begin{array}{c}\text { Buena planeación y coordinación } \\
\text { entres jurisdicciones y } \\
\text { empresarios. }\end{array}$ \\
\hline Espacio público & $\begin{array}{l}\text { Acentúa las zonas privadas (patios, } \\
\text { centros comerciales, clubes privados). }\end{array}$ & $\begin{array}{c}\text { Acentúa el espacio público (vias } \\
\text { públicas, parques, zonas } \\
\text { peatonales). }\end{array}$ \\
\hline
\end{tabular}

Gráfico 7: Modelo de Crecimiento - Plan Maestro de Movilidad, SDM, 2003 


\section{Conclusiones}

- Varias ciudades en el mundo utilizan subsidios para el transporte público de los ciudadanos, cada una de ellas manejando diferentes estrategias y necesidades esto dependiendo de la estructura, movilidad, sociedad y sus dirigentes que son las personas encargadas de mejorar las herramientas puestas por el gobierno en cada gobierno. Queda claro entonces que, volver a implementar esta estrategia en Colombia específicamente Bogotá sería de gran ayuda para mejorar en cierta medida el transporte público en la capital, logrando con esto más accesibilidad e igualdad para todo tipo de personas que necesiten o quieran utilizar dicho servicio.

- España y Colombia son países que difieren en muchos aspectos en especial en movilidad dado que ellos son un país desarrollado y nosotros subdesarrollado, esto hace que la cultura, las políticas, la economía tengan diferencias significativas como para compararnos o tratar de copiar el modelo. Entendiendo el contexto, lo que podemos hacer es poner en práctica la organización del sistema de movilidad, su cultura organizada con sentido de pertenencia hacia su país y su sociedad que trabajan y se ayudan entre todos.

- Finalmente, esta investigación estuvo basada en varios puntos de vista como lo son estado actual, infraestructura y social, pero a medida que fuimos desarrollando y describiendo el tema fuimos encontrando que una de las claves para mejorar la movilidad no se basa solamente en estructuras sino también en acoger a todos los ciudadanos para que el transporte sea accesible e igualitario para todos. No sin ser más importante, para poder lograr una mejoría en la movilidad de Bogotá y que el transporte sea de calidad es necesario que desde el gobierno se piense y se actué de manera igualitaria y trace una organización en la estructura del transporte ya que es una oportunidad fundamental ahora Transporte Publico en Bogotá y Madrid 24 con el metro para Bogotá idea que se estructura a medida que pasa el tiempo, ojalá para esta inversión se tengas muchos más aspectos que con el actual medio de transporte.

- Si bien sabemos que los 2 sistemas están pensados de forma distinta, para ciudades distintas. Los 2 esta orientados a ofrecer un sistema integrado. Sin embargo, aparte de la planeación del modelo de transporte esta la trazabilidad y la evolución de este dependiendo de las necesidades que experimente cada ciudad. En este caso es donde surge una brecha representativa en los modelos ya se vuelven obsoletos si no se tiene evolución constante perjudicando a la mayoría de la población que toma transporte púbico.

- Son muchos los inconvenientes que actualmente posee Transmilenio que, si bien es una ciudad en la que la flota de buses no da abasto, se pueden implementar un cambio en el funcionamiento como lo propuso el profesor Guillermo Ramírez al decir que la falla en el sistema se centra en la variedad de rutas innecesarias que circulan en la ciudad y es por esto que unas las vemos a su 
máxima capacidad y otras vacías. Al haber tantas rutas estas a su vez entorpecen las paradas y la circulación de estas, generando de igual manera altos costos que se trasladan al consumidor. Teniendo esto claro, la propuesta es generar 2 rutas por portal, una expresa y otra como ruta fácil, que permita ser el bus que le funcione a todo independiente de para donde vaya, de esta manera se reducen trayectos de viaje, flota y reducción de costos.

- La cultura refleja lo que somos, si bien hay muchas cosas que mejorar y tomar como ejemplo de los demás transportes alrededor del mundo. También en cierto que nosotros somos el principal beneficiario, es por esto por lo que al tener más tolerancia y pensar en el beneficio general y no en el particular, se mejorara a calidad del servicio. Transporte Publico en Bogotá y Madrid 25. 


\section{Referencias}

Duarte Vega, C. (2015). Movilidad urbana: ¿cómo definir un sistema de transporte público colectivo (SITP), en Bogotá,D.C. Colombia. Cuadernos Latinoamericanos de Administración, XI (21), 71-82.

Márquez, L. (2016). La percepción de seguridad en la demanda de transporte de la integración bicicleta-metro en Bogotá, Colombia. Lecturas de Economía, (84), 143-177.

Acevedo, J. (2009). Resumen del libro: El transporte como soporte al desarrollo de Colombia. Una visión al 2040. Revista de Ingeniería, (29), 156-162.

Bermeo Muñoz, E., \& Calderón Sotero, J. (2009). Diseño de un modelo de optimización de rutas de transporte. El Hombre y la Máquina, (32), 52-67.

Bermeo Muñoz, E., \& Calderón Sotero, J. (2009). Diseño de un modelo de optimización de rutas de transporte. El Hombre y la Máquina, (32), 52-67.

Sánchez Rojas, C. (2016). Colombia en el post-acuerdo y el rol de la infraestructura de transporte. Revista de Ingeniería, (44), 28-35.

Cabrera-Moya, D. (2017). Hacia un marco de referencia para la generación de ventajas competitivas sostenibles en los Sistemas Integrados de Transporte Público -SITP en Colombia. Aporte de las capacidades dinámicas de innovación. Dyna, 84 (200), 169-175.

Alirio Valencia, E., \& Poveda, Y., \& Escudero, C. (2012). Breve apología al transporte paralelo. Scientia Et Technica, XVII (50), 170-175.

Hidalgo, D. (2005). Comparación de Alternativas de Transporte Público Masivo - Una Aproximación Conceptual. Revista de Ingeniería, (21), 94-105.

Duarte Forero, E. (2011). El Transporte Público Colectivo en Bogotá, D.C.: Una Mirada desde la Dinámica de Sistemas. Ingeniería, 16 (2), 18-34.

Ortiz Triviño, J., \& Serrano Rivera, L. (2006). Simulación de sistemas de transporte público masivo. Ingeniería e Investigación, 26 (1), 51-57.

Suarez Florez, M. (2001). Los sistemas inteligentes de transporte ITS . Ciencia e Ingeniería Neogranadina, (10), 3945.

Ardila Gómez, A. (2005). La olla a presión del transporte público en Bogotá. Revista de Ingeniería, (21), 56-67.

Urbano, P., \& Ruiz Rúa, A., \& Sánchez Gutiérrez, J. (2012). EL SISTEMA DE TRANSPORTE PÚBLICO EN ESPAÑA: UNA PERSPECTIVA INTERREGIONAL. Cuadernos de Economía, XXXI (58), 195-228.

Rojas Parra, F., \& Mello Garcias, C. (2005). El transporte público colectivo en Curitiba y Bogotá. Revista de Ingeniería, (21), 106-115. 
Jolonch Palau, J. (2013). Análisis del transporte masivo y la movilidad en Bogotá. Universidad \& Empresa, 15 (24), $15-23$.

Pedro Rollan, (2015). Informe Anual Consorcio Regional Transporte de Madrid. Ministerio de Fomento. 